\title{
Pregabalin: A Drug Useful in the Management of Pain after Craniotomy?
}

\author{
Andrea Gentili ${ }^{1}$ \\ ${ }^{1}$ Department of Anaesthesia and Intensive Care, Villa Laura \\ Hospital, Bologna, Italy
}

J Neurosci Rural Pract 2019;10:646-647

In the last years, postoperative analgesia has made significant progress as new drugs and new pharmacological techniques have emerged. Progressively, these acquisitions have also critically reconsidered the established habit of treating postoperative pain only with nonsteroidal anti-inflammatory drugs or opioids. ${ }^{1}$

Multimodal analgesia involves the use of multiple, simultaneous mechanisms of pain control acting synergistically to improve analgesic effect and reduce the doses of any single drug to minimize risks of side effects. ${ }^{2}$

Closely related to multimodal analgesia, the concept of obtaining a better efficacy of postoperative analgesia has become increasingly more important, aiming above all at the specific type of surgery and the profile of the individual patient.

In this issue of the Journal of Neurosciences in Rural Practice, the authors present a topic that is part of this new and interesting line of research. ${ }^{3}$ It proposes a randomized, double-blind, placebo-controlled trial of notable relevance and importance in both cultural clarification and practical clinical use: the effect of pregabalin on postcraniotomy pain in patients undergoing supratentorial tumor surgery.

Pregabalin belongs, together with gabapentin, to the class of drugs called gabapentinoids, originally designed as analogs of gamma-aminobutyric acid, which exert their analgesic effect through the mechanism of the bind to the $\alpha 2$ $\delta-1$ and $\alpha 2 \delta-2$ auxiliary subunits of calcium channels. Such action results in a reduction in the depolarization-induced influx of calcium; hence, there is a reduction in the release of excitatory neurotransmitters including glutamate, noradrenaline, dopamine, and serotonin. ${ }^{4}$

Compared with gabapentin, pregabalin is considered more potent, is associated with fewer adverse effects, is more effective as it is absorbed more quickly, and shows higher biological activity.

The study presented by the authors in this issue starts from two fundamental assumptions: the awareness that very often, the analgesic therapy after craniotomy is insufficient,
Address for correspondence Andrea Gentili, Via Muratori, 3, 40054 Budrio, Bologna, Italy (e-mail: andrea_gentili@libero.it).

and the need to use an analgesic strategy suitable for neurosurgery.

Paradoxically, the organ that is the center of pain and its related mechanisms receives little attention to alleviate distress during neurosurgical procedures. From 30 to $90 \%$ of patients who reported postcraniotomy pain, the majority occurred in the first 48 hours after surgery. ${ }^{5}$

The literature indicates that a valid postoperative analgesic strategy in this particular type of surgery must comply with some of the following criteria ${ }^{6}$ :

- The use of appropriate drugs to allow as much as possible an assessment of neurological function.

- The administration of effective drugs to avoid side effects related to the permanence of pain, such as very harmful hemodynamic fluctuations in patients with impaired cerebral autoregulation.

- The use of pharmacological techniques as free as possible from side effects such as bleeding, postoperative nausea and vomiting, respiratory depression, visual disturbances, dizziness, and neuropsychiatric manifestations, which can affect or hinder monitoring of the neurological examination.

- The employment of drugs with pharmacokinetic handling characteristics such as oral administration and short or intermediate half-life.

These features suggest that pregabalin, chosen in the protocol of this study and long used in the treatment of neuropathic pain, could be proposed in the analgesic strategy after neurosurgery, in which experiences are still scarce.

The authors of the experience proposed in the current issue compare three groups of patients receiving oral placebo, pregabalin $75 \mathrm{mg}$, or pregabalin $150 \mathrm{mg}$ before the induction of anesthesia, respectively. The result of the study is highly significant as it allows us to establish the threshold dose of $150 \mathrm{mg}$ as effective, safe, with few sedation consequences, and with opioid-sparing effects. Doses as low as $75 \mathrm{mg}$ give poor analgesic results. It should be noted that these data are also confirmed in other types of surgery. 
In neurosurgery, another study on the management of pain after craniotomy with pregabalin shows that preoperative use of $150 \mathrm{mg}$ on the evening before surgery, 1.5 hours before entering the operating room, and postoperative twice daily for 72 hours following surgery attenuates preoperative anxiety, improves sleep quality, reduces postoperative pain scores, and reduces analgesic usage without increasing the rate of adverse effects. ${ }^{8}$ It should be stressed that the approach of this study, compared with that proposed in the current issue, differs above all in the daily dose and duration of administration of pregabalin. This condition must, however, be assessed, since in other types of surgery, the administration of pregabalin at doses higher than $150 \mathrm{mg}$ /day or extended to the early postoperative days can cause an increase in sedation; this is considered a potential danger in neurosurgery.

In conclusion, postoperative pain management after craniotomy needs to be effective and safe. it must avoid both the harmful effects of pain on brain cells and the side effects of drugs that can distort or harm the monitoring of the neurological examination. Pregabalin administered preoperatively may have a very important role in analgesic therapy. Further studies may serve to determine the risk/benefit ratio in being able to continue administration in the postoperative period.

\section{Funding}

None.

\section{Conflict of Interest}

None declared.

\section{References}

1 Wick EC, Grant MC, Wu CL. Postoperative multimodal analgesia pain management with nonopioid analgesics and techniques: a review. JAMA Surg 2017;152(7):691-697

2 Tan M, Law LS, Gan TJ. Optimizing pain management to facilitate enhanced recovery after surgery pathways. Can J Anaesth 2015;62(2):203-218

3 Lamsal R, Mahajan C, Chauhan V, Gupta N, Mishra N, Rath GP. Effect of pregabalin on post-craniotomy pain in patients undergoing supratentorial tumor surgery: randomized double-blind, placebo-controlled trial. J Neurosci Rural Pract 2019;10:641-645

4 Lam DM, Choi SW, Wong SS, Irwin MG, Cheung CW. Efficacy of pregabalin in acute postoperative pain under different surgical categories: a meta-analysis. Medicine (Baltimore) 2015;94(46):e1944

5 Flexman AM, Ng JL, Gelb AW. Acute and chronic pain following craniotomy. Curr Opin Anaesthesiol 2010;23(5):551-557

6 Lai LT, Ortiz-Cardona JR, Bendo AA. Perioperative pain management in the neurosurgical patient. Anesthesiol Clin 2012;30(2):347-367

7 Sebastian B, Talikoti AT, Nelamangala K, Krishnamurthy D. Effect of oral pregabalin as preemptive analgesic in patients undergoing lower limb orthopedic surgeries under spinal anaesthesia. J Clin Diagn Res 2016;10(7):UC01-UC04

8 Shimony N, Amit U, Minz B, et al. Perioperative pregabalin for reducing pain, analgesic consumption, and anxiety and enhancing sleep quality in elective neurosurgical patients: a prospective, randomized, double-blind, and controlled clinical study. J Neurosurg 2016;125(6):1513-1522 